\title{
Modeling variation in empathic sensitivity using go/no-go social reinforcement learning
}

Katherine O’Connell ${ }^{1 *}$, Marissa Walsh ${ }^{2}$, Brandon Padgett ${ }^{2}$, Sarah Connell ${ }^{2}$ \& Abigail A. Marsh ${ }^{2}$

${ }^{1}$ Interdisciplinary Program in Neuroscience, Georgetown University, Washington, DC, 20057

${ }^{2}$ Department of Psychology, Georgetown University, Washington, DC, 20057

Corresponding author e-mail: kmo52@georgetown.edu 


\section{Abstract} Empathic experiences shape social behaviors and display considerable individual variation.

3 Recent advances in computational behavioral modeling can help rigorously quantify individual

4 differences, but remain understudied in the context of empathy and antisocial behavior. We

5 adapted a go/no-go reinforcement learning task across social and non-social contexts such that

6 monetary gains and losses explicitly impacted the subject, a study partner, or no one. Empathy was

7 operationalized as sensitivity to others' rewards, sensitivity to others' losses, and as the Pavlovian

8 influence of empathic outcomes on approach and avoidance behavior. Results showed that 61

9 subjects learned for a partner in a way that was computationally similar to how they learned for

10 themselves. Results supported the psychometric value of individualized model parameters such as

11 sensitivity to others' loss, which was inversely associated with antisociality. Modeled empathic

12 sensitivity also mapped onto motivation ratings, but was not associated with self-reported trait

13 empathy. This work is the first to apply a social reinforcement learning task that spans affect and

14 action requirement (go/no-go) to measure multiple facets of empathic sensitivity.

23 Keywords: Social learning; empathy; antisociality; behavioral modeling; reinforcement learning 


\section{Introduction}

Empathic responding to the emotions of others - including representing, learning about, and responding to others' outcomes - may be disrupted in populations with antisocial traits and

27 behaviors (Blair, 2001, 2018; Decety et al., 2009; Lozier et al., 2014). The psychological and computational mechanisms underlying empathic responding are important to understand, but thus far remain opaque. Decades of research have shown how the anticipation of reward or punishment for the self can potentiate approach or avoidant behaviors, respectively (Estes \& Skinner, 1941;

31 Rescorla \& Solomon, 1967). But comparatively little is known about how the anticipation of 32 others' affective outcomes influences behavior, or how these processes may be disrupted in 33 individuals with antisocial traits. For this study, we aimed to assess how reinforcement learning, 34 which provides the prevailing framework to describe how organisms learn to obtain rewards and avoid punishments for themselves, can be extended to the social domain to help understand how we learn to make choices that result in outcomes for another person. This social reinforcement learning, or learning for, approach permits quantitative investigation of the influence of empathic processes involved in the first-hand experience of the target emotion (Bernhardt \& Singer, 2012; Decety \& Sommerville, 2003). For example, both winning money and viewing another person win money is associated with neural responses in ventral striatum (Burke et al., 2010; Fareri et al., 2012; Mobbs et al., 2009) and overlapping amygdala activation is observed during both experienced and empathic fear of shock (Lindström et al., 2018), suggesting similar neural processes. Shared responses to experienced and empathic events are also relevant for learning in social contexts. Neural reward prediction errors (i.e., modulations in activity based on the 
47 difference between actual and expected reward outcomes) are observed in ventral striatum 48 regardless of whether subjects learn for their own or a partner's monetary gain (Lockwood et al., 49 2016). Striatal prediction errors are also engaged when learning to avoid a shock to oneself or a 50 partner (Lengersdorff et al., 2020). Both neural prediction error signals (Lockwood et al., 2016) 51 and individualized model parameters that describe heightened sensitivity to others' outcomes52 including learning rate (Lockwood et al., 2016) and value sensitivity (Lengersdorff et al., 2020) 53 have been positively associated with self-reported empathic abilities. This suggests that individual 54 variation in learning when outcomes for another person are at stake reflects empathic traits. 55 However, to our knowledge, no prior study has investigated links between learning across both

rewarding and aversive social outcomes and antisociality.

We thus created a novel go/no-go social reinforcement learning paradigm in which monetary outcomes impacted the subject (the learner), a stranger (introduced to subjects as their study partner) or no one. Subjects learned to perform a go or a no-go action on trials associated with either win or loss, including two congruent trial types (go to win, no-go to avoid loss) and two incongruent trial types (no-go to win, go to avoid loss). This design capitalizes on the inflexibility of conserved, spontaneous behavioral responses to affective states-known as Pavlovian biases. In general, behavioral avoidance is facilitated in anticipation of aversive stimuli, whereas approach is facilitated during anticipation of appetitive stimuli (Chen \& Bargh, 1999; Gray \& McNaughton, 2000; Hershberger, 1986; Lang et al., 1990; Solarz, 1960). This fullycrossed design between action (go/no-go) and valence (win/lose) enables quantification of Pavlovian biases (Guitart-Masip et al., 2014; Guitart-Masip et al., 2012a; 2012b) and provides a deeper understanding of affect's influence on behavior than standard reinforcement learning designs, which typically require a go action (approach) on every trial and frequently assess only 
one valence direction. Our design enabled us to quantify outcome sensitivities and Pavlovian

71 biases for both positively and negatively valenced outcomes and to investigate whether people

72 learn to obtain rewards and avoid punishments for others in a way that is computationally similar

73 to how they learn for themselves.

Consistent with the shared representations view of empathy, we anticipated similarities in learning parameters for the self and social conditions. We also tested whether parameters indexing empathy during the task were associated with trait antisociality and empathy. We hypothesized a negative association between antisociality and modeled empathic sensitivity, particularly for others' loss. In the real-world, impaired empathy for others' distress is posited to be a primary factor contributing to maladaptive antisocial behaviors that cause harm to others' welfare directly or indirectly (Blair, 1995). Because we used a mixed-gender community sample, we focused on social aggression, which is also known as indirect or relational aggression (Archer \& Coyne, 2005). This is a common form of antisocial behavior (Björkqvist et al., 1994; Burt et al., 2012) employed at relatively similar rates by females and males (Archer, 2004; Björkqvist et al., 1994; Burt et al., 2012; Czar et al., 2011; Eagly \& Steffen, 1986; Slawinski et al., 2018), which involves the use or manipulation of social relationships as a means of harming others psychologically or detrimentally affecting their social status (Archer \& Coyne, 2005; Burt \& Donnellan, 2010).

87 Examples include bullying, social exclusion, and spreading rumors. Like other forms of antisociality, social aggression is believed to reflect reduced empathic sensitivity (Decety, 2010; Marsh, 2016, 2018; Miller \& Eisenberg, 1988), and, unlike physical aggression, is well distributed in community samples. However, few studies have applied computational modeling or

91 reinforcement learning to quantify empathic deficits in social aggression. Lastly, and because 92 measures of trait empathy have previously been linked to learning parameters for obtaining a 
93 partner rewards (Lockwood et al., 2016) and avoiding a partner's shock (Lengersdorff et al., 94 2020), we hypothesized a positive association between trait empathy and model parameters 95 capturing variation in empathy for both positive and negative social outcomes.

96

97

98

99

100

101

102

103

104

105

106

107

108

109

110

111

112

113

114

115

\section{Methods}

\section{Subjects}

A sample size of $\mathrm{n}=60$ was predetermined following a power calculation using previously reported data (Lockwood et al., 2016) that observed a positive correlation $(\mathrm{r}=.44)$ between trait empathy and social reinforcement learning rate, for which we anticipated a similar effect size (power $=.80$, alpha $=.05 / 8$ ). We thus enrolled 71 community adults from the Washington, DC metro area in testing (recruitment sources included the ResearchMatch database, flyers, word of mouth, and Georgetown University's Research Volunteer Program that granted course/extra credit to undergraduate students) to yield our final sample ( $\mathrm{n}=61 ; 40 \mathrm{f}, 21 \mathrm{~m} ; 23.0 \pm 6.9$ years old) that excluded 2 subjects who did not complete the study, 5 who responded to $\geq 90 \%$ of trials with the same action, and 3 who stopped responding to the task for $\geq 6$ consecutive blocks. Six additional subjects expressed significant doubt that the social condition would result in real monetary payout to the partner, but were included in the final sample with a coded variable for post-hoc evaluation. Characteristics of the final sample are reported in Supplementary Table 1. Data was collected in 2017 and 2018 and all study procedures were carried out in accordance with a protocol approved by the Institutional Review Board at Georgetown University in Washington, DC, and subjects provided written informed consent prior to testing.

15 Procedure 
A go/no-go reinforcement learning task (Guitart-Masip et al., 2012a; 2012b), which

117 harnesses the interaction between action (go, no-go) and valence (reward, punishment) was

118 adapted to include social and non-social conditions. Subjects first completed paper and pencil

119 forms that included information regarding demographics. Subjects were then briefly introduced to

120 a stranger described to them as their study partner, who was actually a trained female confederate.

121 Subjects did not otherwise interact with this stranger and were told that they would leave at 122 separate times to help minimize social desirability biases.

Subjects were introduced to the paradigm through written and verbal instruction and 124 practiced the task. Subjects were instructed to learn the correct action for each image, either a 125 button press or no button press, and that points would be converted to real money at the end of the 126 task. Each trial consisted of presentation of a fractal image for $1000 \mathrm{~ms}$ followed by a $250 \mathrm{~ms}$ 127 fixation then a $1000 \mathrm{~ms}$ decision screen (Fig. 1). The decision screen presented a white circle on 128 either the right or left side of a central fixation cross, and subjects were told to match button press 129 responses to the circle side using the right or left arrow key if they chose to respond. After another $130250 \mathrm{~ms}$ fixation screen, a $1000 \mathrm{~ms}$ feedback screen showed a green up-arrow for win, a red down131 arrow for loss or a yellow horizontal bar for no change. Each fractal image was associated with 132 only one possible affective valence - that is each image was associated with either: 1) win for 133 correct answers and no change for incorrect answers, or 2) no change for correct answers and loss 134 for incorrect answers. This enabled each image to be tethered to either wins or losses, but not both, 135 resulting in discrete affective mapping. The mapping between action requirement and affect was 136 orthogonalized such that win-associated cues sometimes required no-go responses while loss137 associated cues sometimes required go responses, creating two difficult trial types and two easy 138 trial types (Pavlovian biases render go to win easier than go to avoid loss and no-go to avoid loss 
139 easier than no-go to win). Subjects were not made aware of this interaction and were not told that

140 fractal images were discretely associated with either possible win or loss. Outcomes were

141 probabilistic such that the correct feedback was provided $80 \%$ of the time, while the alternate

142 feedback was randomly provided $20 \%$ of the time.

143 The three condition types were always explicitly known to subjects; each condition block

144 was introduced by a 4 second screen that signaled the condition type ("You are now playing for

145 [YOURSELF] / [YOUR PARTNER] / [NO ONE]"') and was associated with a specific border

146 color presented throughout the condition block. Three $120 \mathrm{~s}$ computerized break periods were

147 built into the paradigm, which split the task into 4 chunks of 9 alternating condition blocks. The

148 task consisted of 720 total trials (240 for each condition, 60 for each trial type, 20 trials within

149 each condition block). Subjects and their partners were separately endowed with $\$ 5$ at the

150 beginning of the task, which subjects could lose from or add to, up to $\$ 10$. Subjects were

151 physically given $5 \$ 1$ bills to increase the saliency and reinforce that real money was at stake.

152 The experimental task was adapted from resources made available from a prior study (Mkrtchian

153 et al., 2017) and was programed in Psychtoolbox 3 (http://psychtoolbox.org).

a

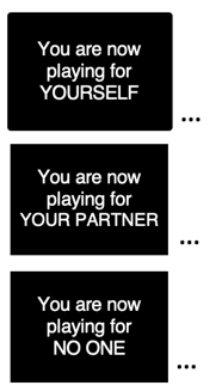

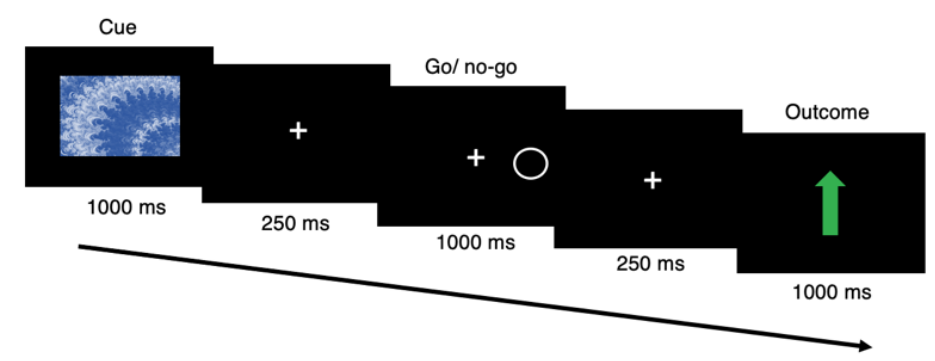

b

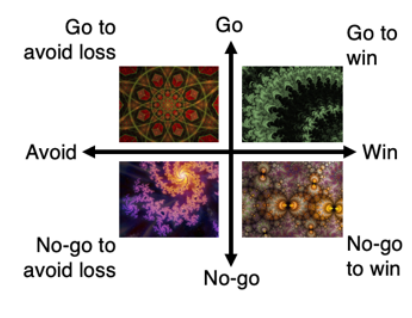

155 Fig. 1 Task Design. (a) Subjects learned a go/no-go reinforcement learning paradigm in three 156 conditions, for when monetary outcomes impacted themselves, their study partner, or no one. On

157 each trial, a fractal image cue was followed by a go/no-go decision screen. Subjects had to learn 
158 the correct response, either a button press (go) or no button press (no-go) over time based on 159 outcome feedback (win, loss, no change). Condition blocks alternated and were signaled by an 160 instruction screen (e.g. "you are now playing for your partner"). (b) All four trial types were 161 present in each condition and were cued by distinct fractal images

162

Following completion of the task, subjects completed a computerized survey that included ratings of effort and motivation (see Supplementary Materials), the Subtypes of Antisocial Behavior Questionnaire (STAB) (Burt \& Donnellan, 2009), the Interpersonal Reactivity Index (IRI) (Davis, 1983), and the Spielberger Trait Anxiety Inventory (STAI-Y2) (Spielberger, 2010). The IRI is a 28 -item measure that assesses trait empathy and includes two subscales indexing cognitive empathy (Perspective Taking, Fantasy) and two subscales indexing affective empathy (Personal Distress, Empathic Concern). The STAB is a 32-item measure that separately assesses Physical Aggression, Social Aggression, and Rule Breaking by summing subscale items. The scale was developed and validated for use in college and community samples and shows good criterionrelated and ecological validity (Burt et al., 2012; Burt \& Donnellan, 2009, 2010). The STAI-Y2 is a 20-item measure of trait anxiety developed for use in community and clinical samples. Four subjects did not complete motivation ratings and 1 subject did not complete the STAI-Y2.

\section{Reinforcement learning models}

All models were adapted from the classic Rescorla-Wagner model (Rescorla \& Wagner, 1972) and were similar to previously reported adaptations used for this go/no-go reinforcement task design (Guitart-Masip et al., 2011; Guitart-Masip et al., 2012b; Mkrtchian et al., 2017). The models, which examine trial-by-trial behavior, use an update rule to calculate action weight on 
181 each trial, Weight $_{a, s}$, allowing for learning about the best action $(a)$ for each trial type $(s)$ over time.

182 Outcomes, $r$, were coded as $+1,0$, or -1 , based on whether the subject won, stayed the same, or

183 lost points, respectively, as provided by feedback to the subject after each decision. This adapted

184 Rescorla-Wagner model multiplies $r_{\text {actual }}$ by a sensitivity parameter, $\beta$, to allow for variations in

185 the effective size of the reinforcement, then subtracts from this product the learned expected

186 outcome resulting in a prediction error. Learning occurs over time by multiplying prediction errors

187 by a learning rate, $\alpha$, and consequently updating the action weight from the preceding trial by

188 adding the new learning (Equation 1). The update rule was calculated separately for each trial type,

$189 S$, meaning that the events occurring between two trials of the same trial type had no influence in

190 the model.

191

192

Weight $_{a, s}=$ Weight $_{a, s-1}+\alpha\left(\left(\beta * r_{\text {actual }}\right)-r_{\text {expected }}\right)$

(Equation 1)

193

Action weights were then passed through a softmax function (Sutton \& Barto, 1998)

195 (Equation 2) to obtain action probabilities that introduced an irreducible noise parameter, $\mathrm{x}$,

196 which allowed for added choice noise in the conversion between action weights and action

197 probabilities ( $\mathrm{x}$ was set to 0 for the model without an $\mathrm{x}$ parameter).

198

199 Probability $_{a, s}=\left[\frac{\exp \left(\text { Weight }_{a, s}\right)}{\Sigma \exp \left(\text { Weight' }_{a, s}\right)}\right](1-\mathrm{x})+\frac{\mathrm{x}}{2}$

(Equation 2)

Incremental modifications (Table 1) to the Rescorla-Wagner model included the following 202 additions: 1) b: a constant go bias parameter that added to the go action weight, 2) $\pi$ : the Pavlovian 203 bias parameter which was multiplied by the affective state value (also calculated by a Rescorla- 
204 Wagner update rule), and the product of which was added to the action weight of go for positive 205 affective states and added to the action weight of no-go for negative affective states, 3) q: an initial 206 go bias parameter which set the initial go action weight greater than 0 to account for subjects' 207 initial tendency to perform go actions at the beginning of the task. Finally, additional models 208 evaluated fits when allowing for separate sensitivities to win and loss $\left(\beta_{\text {win, }}, \beta_{\text {loss }}\right)$ and separate

209 Pavlovian bias parameters for win and loss $\left(\pi_{\mathrm{win}}, \pi_{\mathrm{loss}}\right)$. These modifications are highly similar to 210 those described previously (Guitart-Masip et al., 2014; Guitart-Masip et al., 2012b; Mkrtchian et 211 al., 2017).

212

213 Table 1. Reinforcement learning models

Model (number of parameters)
Parameters

\begin{tabular}{|c|c|c|c|c|c|}
\hline $\begin{array}{l}\text { Sensitivity } \\
(\beta)\end{array}$ & $\begin{array}{c}\text { Learning } \\
\text { rate }(\alpha)\end{array}$ & $\begin{array}{l}\text { Choice } \\
\text { noise } \\
\text { (x) }\end{array}$ & $\begin{array}{c}\text { Constant } \\
\text { go bias (b) }\end{array}$ & $\begin{array}{l}\text { Pavlovian } \\
\text { bias }(\pi)\end{array}$ & $\begin{array}{c}\text { Initial go } \\
\text { bias (q) }\end{array}$ \\
\hline 1 & 1 & & & & \\
\hline 1 & 1 & 1 & 1 & & \\
\hline 1 & 1 & 1 & 1 & 1 & \\
\hline 2 & 1 & 1 & 1 & 1 & \\
\hline 1 & 1 & 1 & 1 & 2 & 1 \\
\hline 2 & 1 & 1 & 1 & 2 & 1 \\
\hline
\end{tabular}

217 models were fit to data from the self, social, and no one conditions separately, or a pooled 218 distribution, in which models were fit to the entire dataset treating each condition and each subject 219 as independent. Model comparison and assessment of distributions were analyzed using integrated Bayesian Information Criterion (iBIC) which penalizes models with more parameters. To 
221 quantitatively compare the unpooled distribution model fit to the pooled distribution model fit,

222 iBIC values were summed across the separately modeled self, social, and no one unpooled model

223 fits (Mkrtchian et al., 2017).

224 Estimated parameters were fit for each subject and each condition using hierarchical 225 expectation-maximization within emfit_toolbox code; https://github.com/mpc-ucl/emfit. Briefly, 226 this process iterates between estimating parameters using maximum a posteriori inference and 227 Laplacian approximation given group priors, then subsequently calculating new group mean priors 228 from updated parameter estimates.

\section{Statistical inference}

To investigate within-subject effects, two-tailed repeated measures ANOVA tests 232 evaluated mean accuracy and effect sizes are reported as partial eta squared $\left(\eta p^{2}\right)$ with the 233 corresponding $95 \% \mathrm{CI}$. To test whether subjects performed significantly above chance overall, we 234 applied a two-tailed, one-sample t-test against 50\%; effect size is reported as Cohen's $d$ with the 235 corresponding $95 \% \mathrm{CI}$.

236 To investigate individual differences in model parameters, untransformed parameter 237 estimates of interest from the winning model were Z-scored and submitted to a Pearson correlation 238 test. Significance values for correlation tests $\left(\mathrm{p}_{\text {perm}}\right)$ were obtained using permutation testing 239 implemented in Matlab; all tests were two-tailed and used 10,000 permutations to create a null 240 distribution. Observation of a linear relationship was followed by multiple linear regression 241 controlling for basic demographic variables and the corresponding parameter from the self 242 condition. 


\section{Traditional analyses of behavior}

Mean accuracy was assessed to initially confirm the presence of predicted Pavlovian behavioral biases in the congruent and incongruent trial types that crossed action requirement and valence (Fig. 2). As expected, a three-way condition (self, social, no one) $\mathrm{x}$ action (go, no-go) $\mathrm{x}$ valence (win, avoid loss) repeated measures ANOVA revealed a significant action $\mathrm{x}$ valence interaction $\left(\mathrm{F}(1,60)=32.39, \mathrm{p}<.001, \eta \mathrm{p}^{2}=0.35,95 \% \mathrm{CI}=[.16, .50]\right)$ such that the go action was

potentiated by reward and the no-go action was potentiated by loss. The lack of a three-way 252 interaction indicated that the action by valence interaction was similarly present across conditions $(F(2,120)=0.31 p=.733)$. A main effect of condition $\left(F(2,120)=13.14, p<.001, \eta p^{2}=.18,95 \%\right.$

$254 \mathrm{CI}=[.06, .29])$ and post-hoc testing with Bonferroni correction revealed that mean accuracy was 255 significantly reduced in the social condition $(\mathrm{M}=55.36 \%, \mathrm{SD}=1.29 \%, 95 \% \mathrm{CI}=[52.78 \%, 57.94 \%])$ relative to the self $(\mathrm{M}=60.68 \%, \mathrm{SD}=1.44 \%, 95 \% \mathrm{CI}=[57.80 \%, 63.57 \%] ; \mathrm{p}<.001)$ and no one $257(\mathrm{M}=58.36 \%, \mathrm{SD}=1.16 \%, 95 \% \mathrm{CI}=[56.04 \%, 60.69 \%] ; \mathrm{p}=.019)$ conditions; no statistically significant difference emerged between accuracy in the self and no one conditions $(p=.069)$. indicated subjects were more accurate on go trials $(\mathrm{M}=70.78 \%, \mathrm{SD}=2.03 \%, 95 \% \mathrm{CI}=[66.73 \%$,

$26174.84 \%])$ relative to no-go trials $(\mathrm{M}=45.48 \%, \mathrm{SD}=2.17 \%, 95 \% \mathrm{CI}=[41.15 \%, 49.82 \%])$ and a 262 significant main effect of valence $\left(\mathrm{F}(1,60)=16.57, \mathrm{p}<.001, \eta \mathrm{p}^{2}=0.22,95 \% \mathrm{CI}=[.06, .38]\right)$ indicated 263 subjects were more accurate on avoid loss trials $(\mathrm{M}=61.19 \%, \mathrm{SD}=1.39 \%, 95 \% \mathrm{CI}=[58.42 \%$, 264 $63.97 \%])$ than win trials $(\mathrm{M}=55.08 \%, \mathrm{SD}=1.37 \%, 95 \% \mathrm{CI}=[52.34 \%, 57.82 \%])$. Subjects' overall

265 accuracy was significantly above chance level $(\mathrm{M}=58.14 \%, \mathrm{SD}=9.03 \%, 95 \% \mathrm{CI}=[55.82 \%$, 266 $60.45 \%] ; \mathrm{t}(60)=7.04, \mathrm{p}<.001, \mathrm{~d}=0.90,95 \% \mathrm{CI}=[0.60,1.20])$. The number of trials in which 
267 subjects pressed the incorrect arrow key (right or left depending on circle side) accounted for very 268 few trials $(\mathrm{Mdn}=4$ out of 720 total trials, $\mathrm{IQR}=6.5)$.

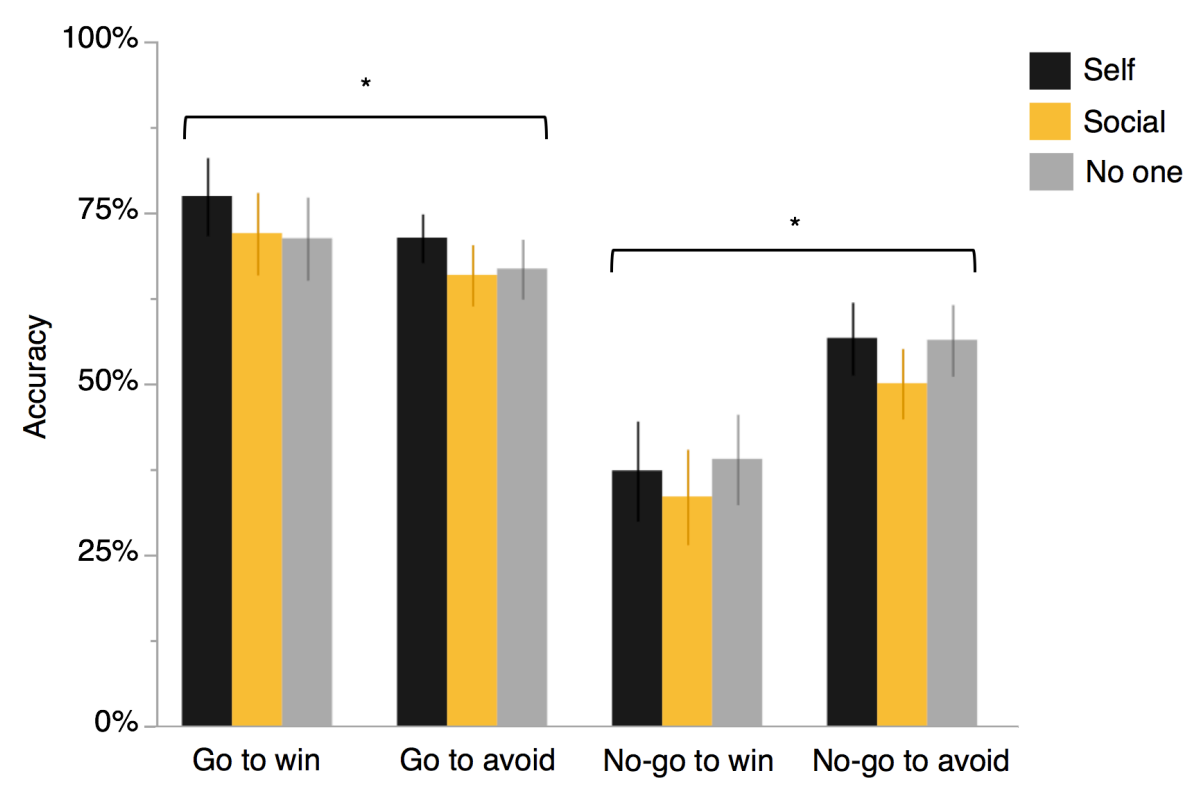

271 Fig. 2 Overall performance accuracy. A significant action $x$ valence interaction indicated that

272 learning was impaired when action requirement was incongruent with valence (e.g. when no-go

273 was required to win and when go was required to avoid loss). A main effect of condition

274 indicated that accuracy was lowest for the social condition. Error bars represent 95\% CI.

\section{Winning model and validation}

Models were compared for fit at the group level using iBIC, which penalizes models with

278 more parameters. The winning model $(2 \beta \alpha \mathrm{xb} 2 \pi \mathrm{q})$ contained 8 parameters, which included one 279 constant learning rate $(\alpha)$, separate sensitivities for rewards and punishments $\left(\beta_{\text {win }}, \beta_{\text {loss }}\right)$, choice 280 noise (x), a constant go bias (b), two separate Pavlovian bias parameters for wins and losses $\left(\pi_{\mathrm{win}}\right.$, $281 \pi_{\text {loss }}$ ), and an initial go bias $(q)$. All models tested showed better performance when using the 
282 pooled distribution (Supplementary Fig. 1), which fit data from all conditions in the same series 283 of expectation-maximization steps, rather than fitting entirely separately for each condition. The 284 pooled $2 \beta \alpha \mathrm{xb} 2 \pi \mathrm{q}$ model provides separate parameters for each subject and each condition. 285 Transformed (Guitart-Masip et al., 2012b; Mkrtchian et al., 2017) parameters from the winning 286 pooled model are plotted in Supplementary Fig. 2. Note that when modeling data separately by 287 condition, the same $2 \beta \alpha \times \mathrm{xb} 2 \pi \mathrm{q}$ winning model performed best for both the self (iBIC $\left.=1.50 \times 10^{4}\right)$ 288 and social $\left(\mathrm{iBIC}=1.55 \times 10^{4}\right)$ conditions; data collected during the no one condition was slightly 289 better fit by the 7 parameter model $\left(\beta \alpha \mathrm{xb} 2 \pi \mathrm{q} ; \mathrm{iBIC}=1.58 \times 10^{4}\right)$, in which the same $\beta$ parameter 290 value was used for both wins and losses. Together, these findings support the prediction that 291 subjects learn to obtain rewards and avoid punishments for others in a way that is computationally 292 similar to how they learn for themselves.

The winning $2 \beta \alpha \mathrm{xb} 2 \pi \mathrm{q}$ model and estimated parameters for each subject were passed 294 through a posterior predictive model to simulate behavior. Overall accuracy and learning curves 295 of the simulated data follow closely with the subjects' real data (Fig. 3). The model performed 296 well overall, but similar to a prior report (Mkrtchian et al., 2017), does not fully capture subjects' 297 extremely poor performance on the no-go to win trial type. Plots of actual mean accuracy relative 298 to simulated mean accuracy at the subject level are displayed in Supplementary Fig. 3. 
a

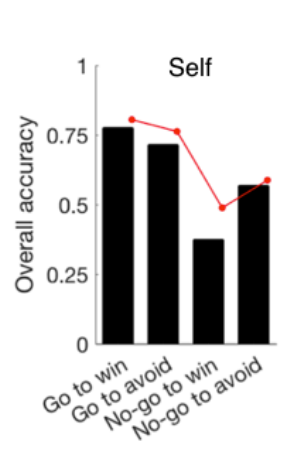

$b$

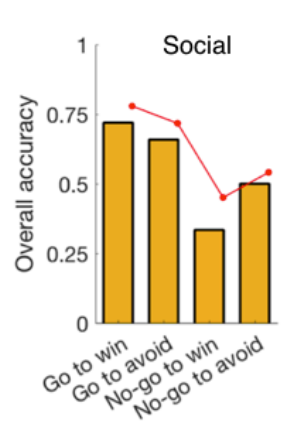

c

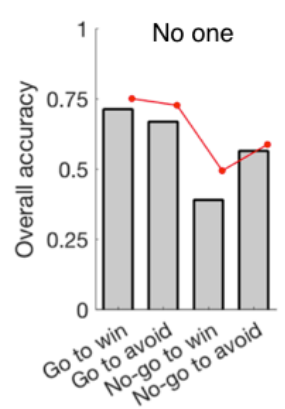

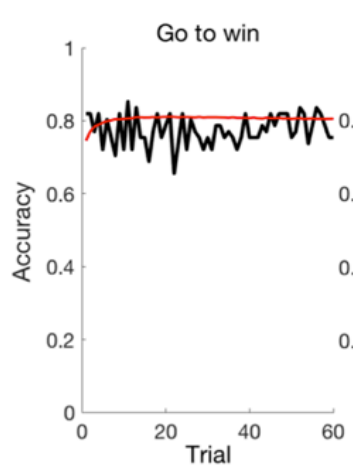
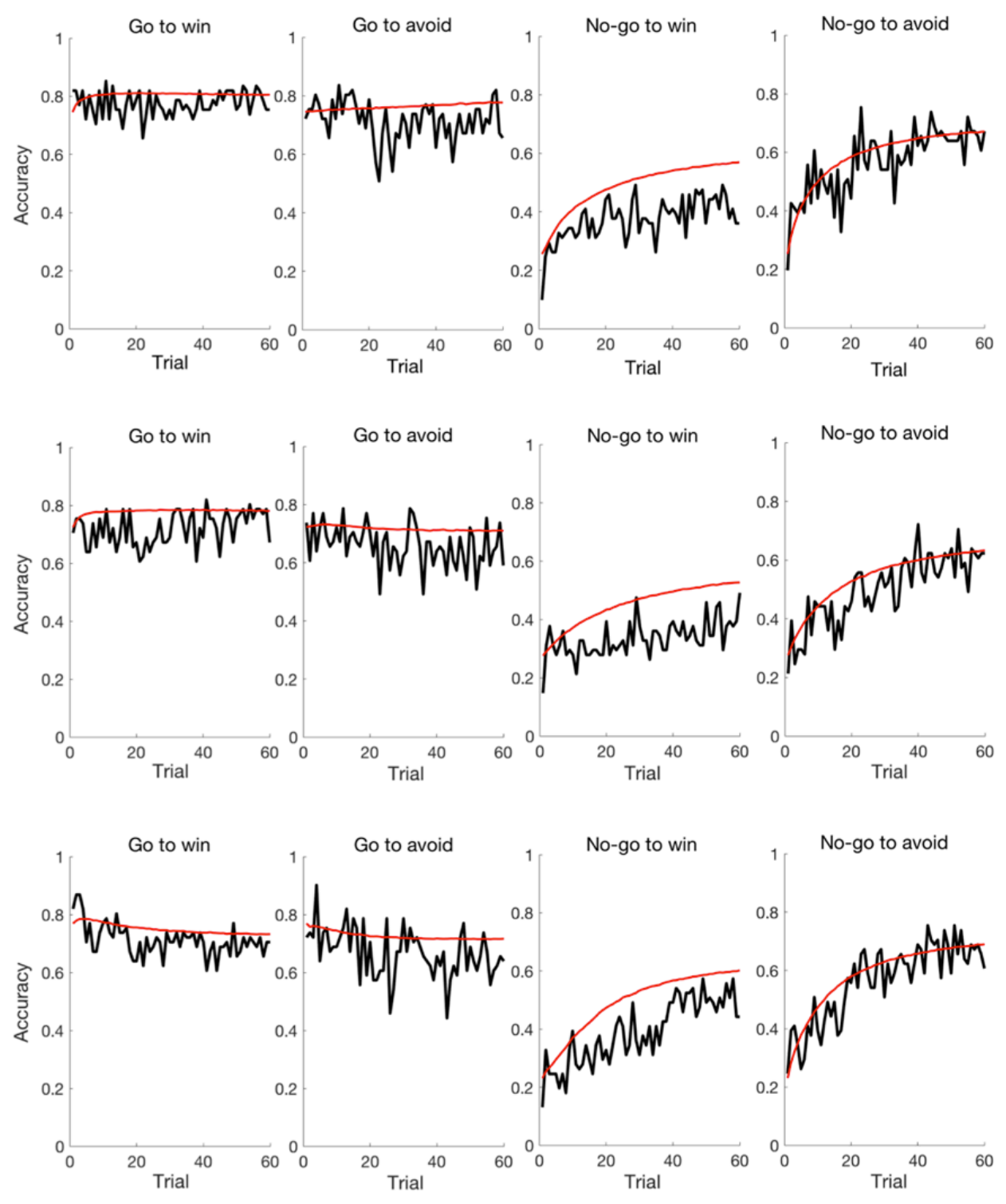

Fig. 3 The posterior predictive model tracks subjects' actual performance. Colored bar

301 graphs display subjects' mean accuracy in the self (a), social (b), and no one (c) conditions in comparison to simulated mean accuracy from the posterior predictive model (red). Line graphs show mean accuracy over time for real data (black) and simulated data (red) 
As hypothesized, bivariate tests found that antisociality was negatively associated with $\beta$ sensitivity to the partners' outcomes. STAB Social Aggression subscale scores were negatively correlated with $\beta$ sensitivity for the partner's loss $\left(\mathrm{r}=-.273, \mathrm{p}_{\text {perm }}=.034\right.$; Fig. $4 \mathrm{~b}$; Supplemental Table 3) and, at a trend level, $\beta$ sensitivity for the partner's win ( $r=-.246, \mathrm{p}_{\text {perm }}=.055$; Fig. $\left.4 \mathrm{a}\right)$. In other words, increased antisociality was associated with reduced impact of the partners' affective 313 outcomes on learning.

314 We next tested whether these relationships were retained after controlling for 315 demographics and the corresponding $\beta$ sensitivity for experienced (self) outcomes using multiple 316 linear regression (Table 2). For the regression model predicting $\beta_{\text {loss }}$ social, the association with 317 antisociality persisted $\left(\beta=-.22, \mathrm{t}(55)=2.06, \mathrm{p}=.044\right.$; model $\left.\mathrm{R}^{2}=.41, \mathrm{p}<.001\right)$ after accounting for 318 age, sex, education level and $\beta_{\text {loss }}$ during the self condition. That is, antisociality remains 319 negatively associated with $\beta$ sensitivity to a stranger's loss even when controlling for $\beta$ sensitivity 320 to experienced loss. Results of the regression model predicting the $\beta_{\text {win }}$ social parameter, however, 321 revealed that antisociality had no significant predictive value when accounting for age, sex, 322 education and the $\beta_{\text {win }}$ self parameter $\left(\beta=-.13, \mathrm{t}(55)=1.16, \mathrm{p}=.252\right.$; model $\left.\mathrm{R}^{2}=.35, \mathrm{p}<.001\right)$. These 323 results suggest that for negative affect, the empathic social condition appears to capture additional 324 information relevant for antisociality beyond what could be explained by self-oriented learning. 325 The relationship between $\beta_{\text {loss }}$ social and antisociality was unaffected by removal of subjects that 326 expressed significant doubt that the social condition would result in real monetary outcomes for 327 the partner $\left(\beta=-.22, \mathrm{t}(49)=2.02, \mathrm{p}=.049\right.$; model $\left.\mathrm{R}^{2}=.46, \mathrm{p}<.001\right)$. Importantly, no significant associations were observed between antisociality and $\beta_{\text {loss }}$ 329 sensitivities obtained during the self $\left(\mathrm{r}=-.127, \mathrm{p}_{\text {perm }}=.328\right)$ or no one $\left(\mathrm{r}=-.206, \mathrm{p}_{\text {perm }}=.111\right)$ 
conditions. Trend level negative associations were observed between antisociality and $\beta_{\text {win }}$ sensitivities obtained during the self $\left(\mathrm{r}=-.247, \mathrm{p}_{\mathrm{perm}}=.054\right)$ and no one $\left(\mathrm{r}=-.223, \mathrm{p}_{\mathrm{perm}}=.084\right)$

332 conditions. Lastly, and further supporting the psychometric value of the social $\beta_{\text {loss }}$ parameter, 333 post-task ratings of motivation to avoid loss in the social relative to self condition positively 334 correlated with this parameter $\left(\mathrm{r}=.361, \mathrm{p}_{\mathrm{perm}}=.006\right.$; Supplemental Fig. 4).
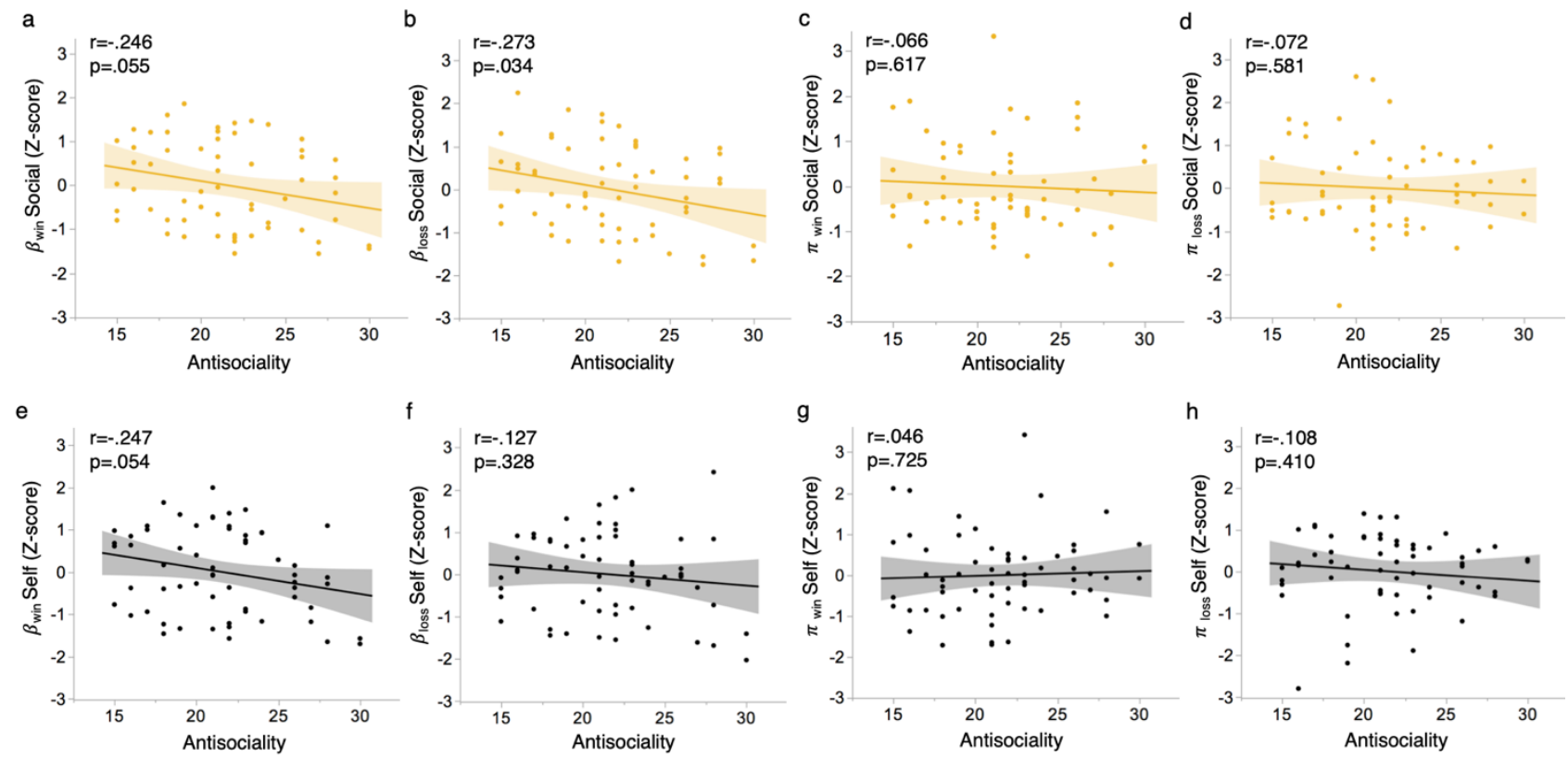
Fig. 4 Correlations between antisociality and model parameters $\beta$ sensitivity and $\pi$

Pavlovian bias. Pearson correlations between z-scored model parameters obtained for the social (a-d) and self (e-h) conditions. Reported significance values are uncorrected and were obtained using two-tailed permutation tests 
345 Table 2. Multiple linear regression models predicting $\beta$ sensitivity for the partners' loss and win.

\begin{tabular}{|c|c|c|c|c|c|}
\hline \multirow[t]{7}{*}{$\beta_{\text {loss }}$ Social $^{\mathrm{a}}$} & & $\mathrm{B}[95 \% \mathrm{CI}]$ & $\beta$ & $\mathrm{t}$ & $\mathrm{p}$ \\
\hline & Constant & $0.60[-0.79,1.98]$ & & 0.86 & .394 \\
\hline & Age & $0.03[-0.01,0.06]$ & .18 & 1.41 & .165 \\
\hline & $\operatorname{Sex}^{\mathrm{c}}$ & $0.13[-0.33,0.58]$ & .06 & 0.55 & .582 \\
\hline & Education $^{\mathrm{d}}$ & $-0.39[-0.98,0.19]$ & -.16 & -1.35 & .184 \\
\hline & $\beta_{\text {loss }}$ Self & $0.50[0.28,0.72]$ & .50 & 4.59 & $<.001^{*}$ \\
\hline & Antisociality & $-0.06[-0.11,-.001]$ & -.22 & -2.06 & $.044 *$ \\
\hline \multirow[t]{7}{*}{$\beta_{\text {win }}$ Social $^{\mathrm{b}}$} & & $\mathrm{B}[95 \% \mathrm{CI}]$ & $\beta$ & $\mathrm{t}$ & $\mathrm{p}$ \\
\hline & Constant & $0.13[-1.36,1.62]$ & & 0.18 & .860 \\
\hline & Age & $0.03[-0.01,0.06]$ & .17 & 1.37 & .175 \\
\hline & $\operatorname{Sex}^{\mathrm{c}}$ & $0.11[-0.37,0.58]$ & .05 & 0.45 & .656 \\
\hline & Education $^{\mathrm{d}}$ & $-0.35[-0.95,0.25]$ & -.14 & -1.17 & .249 \\
\hline & $\beta_{\text {win }}$ Self & $0.52[0.29,0.74]$ & .52 & 4.52 & $<.001 *$ \\
\hline & Antisociality & $-0.03[-0.09,0.02]$ & -.13 & -1.16 & .252 \\
\hline \multicolumn{6}{|c|}{${ }^{\mathrm{a}}$ Model predicting $\beta_{\text {loss }}$ Social, $\mathrm{R}^{2}=.41, \mathrm{p}<.001 .{ }^{\mathrm{b}}$ Model predicting $\beta_{\text {win }}$ Social, $\mathrm{R}^{2}=.35, \mathrm{p}<.001$} \\
\hline \multicolumn{6}{|c|}{ Sex was coded as 1 for female and 0 for male. ${ }^{\mathrm{d}}$ Education was coded as 1 for $\geq 4$-year degree } \\
\hline
\end{tabular}

Contrary to expectations, antisociality did not show a significant relationship with

351 Pavlovian bias parameters $\left(\pi_{\text {win }}\right.$ or $\left.\pi_{\text {loss }}\right)$ during the social or self conditions $\left(p_{\text {perm }} \geq .410\right)$. However,

352 similar to prior literature (Mkrtchian et al., 2017) we observed that high trait anxiety was associated

353 with increased Pavlovian avoidance bias during self-experienced negative affect ( $\mathrm{r}=.258$,

$354 \mathrm{p}_{\text {perm }}=.045$; Supplementary Table 5), providing evidence that the Pavlovian bias parameter 355 successfully captured meaningful socioemotional variance.

In addition, no significant association was observed between self-reported trait empathy

357 and model parameters indexing empathic sensitivity (social $\beta_{\text {win }}$ and $\beta_{\text {loss }}$ ) or empathic Pavlovian 358 bias ( $\operatorname{social} \pi_{\mathrm{win}}$ and $\pi_{\text {loss }}$ ) (IRI Total; all $\mathrm{p}_{\mathrm{perm}} \geq .320$ ). Exploratory assessment of IRI subscales also 
359 showed no significant association with these parameters (all perm $\geq .098$ ). Full statistics are 360 displayed in Supplemental Table 4.

Bivariate correlation tables between social learning model parameters and self-reported

362 traits are displayed in Supplemental Tables 3-5. Of note, antisociality was also positively

363 correlated with social learning rate $\alpha\left(\mathrm{r}=.285, \mathrm{p}_{\mathrm{perm}}=.025\right)$.

\section{Discussion} activation, we found that learning from experienced and empathic outcomes relies on similar computational processes and that individual differences in the weights of these computations meaningfully relate to socioemotional traits, particularly antisociality. Subjects were able to learn

370 a task when monetary outcomes impacted a stranger study partner, but subjects who reported

371 increased antisociality exhibited reduced sensitivity to the partner's outcomes, in line with theories

372 that empathic deficits may lay the foundation for the development of antisocial behavior (Decety, 373 2010; Marsh, 2016, 2018; Miller \& Eisenberg, 1988). Reduced empathic sensitivity for losses was 374 associated with antisociality even after adjusting for demographic variables and self-oriented loss 375 sensitivity, suggesting the social learning condition captured uniquely meaningful information 376 about antisocial traits. To our knowledge, this is the first report to identify a link between 377 antisociality and empathic sensitivity using a social reinforcement learning paradigm. Task ratings 378 of self-reported motivation to avoid loss for the partner were also associated with increased 379 empathic sensitivity for losses, and in line with a prior study (Mkrtchian et al., 2017), trait anxiety 380 correlated with heightened Pavlovian avoidance bias when facing potential loss to the self (but not 
381 the partner). These results indicate the task and modeling are suitable for assessing individual differences across distinct socioemotional traits.

By incorporating go/no-go action requirements, we quantified Pavlovian behavioral

384 biases - the inflexible mappings between behavioral approach/ avoidance and positive/ negative 385 affective states - for both self and social learning. Analyses of overall accuracy and model fitting 386 confirmed the presence of Pavlovian biases across conditions; however, we found no clear link 387 between Pavlovian biases and antisociality. These results point to the utility of our paradigm to 388 separately quantify sensitivity to empathic outcomes $(\beta)$ and the influence of empathic affect on 389 approach and avoidance Pavlovian biases $(\pi)$, with only the former associated with antisociality 390 in our sample. Other paradigms have indicated generally reduced influence of affect on 391 approach/avoidance behavior in violent incarcerated samples (Ly et al., 2016) and impaired 392 inhibition in response to punishment cues during passive avoidance learning in youths with 393 clinical-level conduct problems (White et al., 2014, 2016)—suggesting that variations in the 394 Pavlovian bias parameter may be associated with more extreme forms of antisociality.

395 Many experimental paradigms used to study antisociality measure affective responding 396 only as it applies to outcomes for the self-for example, behavioral, physiological or neural 397 responses to affective events experienced first-hand (Arnett, 1997; Buckholtz et al., 2010, 2017; 398 De Brito et al., 2013; Lorber, 2004; Murray et al., 2017, 2018; White et al., 2014, 2016). But 399 antisocial behaviors are - by definition - fundamentally about disrupted social behaviors that 400 impact other individuals. Thus, understanding antisociality and its computational origins may be 401 better achieved by the techniques we report here and through incorporation of explicitly social 402 (empathic) task conditions. 
While antisociality, trait anxiety and post-task motivation ratings all showed largely expected correlations with model parameters, we observed no significant relationship with selfreported empathy measured using IRI Total Score. These findings contrast with previous reports

406 of increased social reinforcement learning rate $\alpha$ (Lockwood et al., 2016) and choice sensitivity 407 (referred to here as choice noise, x) (Lengersdorff et al., 2020) being associated with heightened 408 self-reported empathy. Both of these previous investigations used the Questionnaire of Cognitive 409 and Affective Empathy — a scale developed by selecting optimal items from prior empathy 410 questionnaires including IRI (Reniers et al., 2011), which may account for the absence of similar 411 results in the present study. The $\alpha$ learning rate parameter from the current investigation may also 412 not be well suited for comparison with learning rates obtained from prior social reinforcement 413 learning designs. Due to the orthogonalization of action and valence in the current study, half of 414 the trial types require that learning overcome affective Pavlovian biases. For example, high 415 sensitivity to partner outcomes in the current investigation was expected to - and did - inhibit 416 learning on the go to avoid loss and no-go to win trials, which may explain why antisociality was 417 correlated with increased social $\alpha$ learning rate but reduced $\beta$ empathic sensitivity. The absence 418 of a relationship between model parameters and self-reported empathy may also reflect the 419 generally poor reliability of self-reported empathy measures, given their poor predictive validity 420 in interpersonal settings (Bernardo et al., 2018; Ickes et al., 2000; Marsh et al., 2007; Vachon et 421 al., 2014) and subjectivity to strong bias and demand characteristics (Zhou et al., 2003). Some additional limitations should be considered in interpreting results. Although subjects 423 met their study partner before the task to increase ecological validity, it remains to be determined 424 whether present results would apply to more complex or face-to-face social situations. In addition, 425 the task operationalized empathic affect as discrete parameters constrained within the framework 
426 of reinforcement learning. Although defining empathy in this stringent way carries strengths, fully

427 understanding empathic affect and its behavioral consequences will require implementing diverse 428 modeling techniques (e.g., drift diffusion, hyperbolic discounting) across various social paradigms 429 and across species (Lockwood et al., 2020).

We obtained relatively small learning rates across conditions (median $\alpha$ values ranged from 431.046 to .053). Although subjects overall performed significantly above chance and our model 432 simulated data well, future investigations - especially those that involve brain imaging — should 433 consider the tradeoff between task complexity and the potential speed of learning, particularly in 434 relation to trial-by-trial signals of interest (e.g. action values). Lastly, the effect sizes observed in 435 the current study were small, suggesting that replication in a large and diverse sample will be 436 important and that future work may benefit from refining the task or methods to increase specificity 437 (e.g., removing the no one condition, which rather than serving as a non-active comparator may 438 capture participation effort or social desirability, which may be related to socioemotional traits of 439 interest).

$440 \quad$ This learning for paradigm appears to aptly model empathic responding for both positive 441 and negative valence, and in combination with go/no-go requirements, aids in understanding how 442 empathic affect influences behavioral approach and avoidance. We provide the first evidence 443 linking antisociality to impaired sensitivity to others' monetary outcomes during learning, 444 supporting the utility of computational learning models for understanding the processes shaping 445 variance in socioemotional traits. 
450 The authors thank Lydia Meena for help with data collection and Shawn A. Rhoads for helpful

451 feedback.

\section{Funding}

454 This work was supported by the National Center for Advancing Translational Sciences of the

455 National Institutes of Health under Award Number TL1TR001431 to KO and by internal

456 Georgetown University funding provided to AAM.

457

458

\section{Conflicts of interest}

459 On behalf of all authors, the corresponding author states that there is no conflict of interest.

\section{Availability of data and material}

462 The datasets generated and analyzed in the current study are available in the Open Science

463 Framework Repository, osf.io/u9fx3/.

464

465

Code availability

466 Code specific to this study is made available osf.io/u9fx3/. The emfit_toolbox code was provided 467 by Q.J.M. Huys and is available at https://github.com/mpc-ucl/emfit.

\section{Authors' contributions}

470 KO conceptualized study, programmed tasks, collected data, analyzed data, and wrote

471 manuscript; MW, BP, and SC collected data; AAM conceptualized study, acquired funding, and 
472 provided critical revisions to the manuscript. All authors approved the final version of the 473 manuscript.

474

475

476

477

478

479

480

481

482

483

484

485

486

487

488

489

490

491

492

493

494

\section{References}

Archer, J. (2004). Sex differences in aggression in real-world settings: A meta-analytic review. Review of General Psychology, 8(4), 291-322. https://doi.org/10.1037/1089-2680.8.4.291

Archer, J., \& Coyne, S. M. (2005). An Integrated Review of Indirect, Relational, and Social Aggression. Personality and Social Psychology Review, 9(3), 212-230. https://doi.org/10.1207/s15327957pspr0903_2

Arnett, P. A. (1997). Autonomic responsivity in psychopaths: A critical review and theoretical proposal. Clinical Psychology Review, 17(8), 903-936. https://doi.org/10.1016/S02727358(97)00045-7

Bernardo, M. O., Cecílio-Fernandes, D., Costa, P., Quince, T. A., Costa, M. J., \& CarvalhoFilho, M. A. (2018). Physicians' self-assessed empathy levels do not correlate with patients' assessments. PLoS ONE, 13(5), e0198488. https://doi.org/10.1371/journal.pone.0198488

Bernhardt, B. C., \& Singer, T. (2012). The Neural Basis of Empathy. Annual Review of Neuroscience, 35(1), 1-23. https://doi.org/10.1146/annurev-neuro-062111-150536

Björkqvist, K., Österman, K., \& Lagerspetz, K. M. J. (1994). Sex differences in covert aggression among adults. Aggressive Behavior, 20(1), 27-33. https://doi.org/10.1002/10982337(1994)20:1<27::AID-AB2480200105>3.0.CO;2-Q

Blair, R. J. R. (1995). A cognitive developmental approach to mortality: investigating the psychopath. Cognition, 57(1), 1-29. https://doi.org/10.1016/0010-0277(95)00676-P

Blair, R. J. R. (2001). Neurocognitive models of aggression, the antisocial personality disorders, 
and psychopathy. Journal of Neurology, Neurosurgery \& Psychiatry, 71(6), 727-731. https://doi.org/10.1136/jnnp.71.6.727

Blair, R. J. R. (2018). Traits of empathy and anger: Implications for psychopathy and other disorders associated with aggression. Philosophical Transactions of the Royal Society B: Biological Sciences, 373, 20170155. https://doi.org/10.1098/rstb.2017.0155

Buckholtz, J. W., Karmarkar, U., Ye, S., Brennan, G. M., \& Baskin-Sommers, A. (2017). Blunted Ambiguity Aversion during Cost-Benefit Decisions in Antisocial Individuals. Scientific Reports, 7, 2030. https://doi.org/10.1038/s41598-017-02149-6

Buckholtz, J. W., Treadway, M. T., Cowan, R. L., Woodward, N. D., Benning, S. D., Li, R., ... \& Zald, D. H. (2010). Mesolimbic dopamine reward system hypersensitivity in individuals with psychopathic traits. Nature Neuroscience. https://doi.org/10.1038/nn.2510

Burke, C. J., Tobler, P. N., Baddeley, M., \& Schultz, W. (2010). Neural mechanisms of observational learning. Proceedings of the National Academy of Sciences of the United States of America, 107(32), 14431-14436. https://doi.org/10.1073/pnas.1003111107

Burt, S. A., \& Donnellan, M. B. (2009). Development and validation of the subtypes of antisocial behavior questionnaire. Aggressive Behavior, 35(5), 376-398. https://doi.org/10.1002/ab.20314

Burt, S. A., \& Donnellan, M. B. (2010). Evidence that the Subtypes of Antisocial Behavior questionnaire (STAB) predicts momentary reports of acting-out behaviors. Personality and Individual Differences, 48(8), 917-920. https://doi.org/10.1016/j.paid.2010.02.021

Burt, S. A., Donnellan, M. B., \& Tackett, J. L. (2012). Should social aggression be considered “antisocial”? Journal of Psychopathology and Behavioral Assessment, 34(2), 153-163. https://doi.org/10.1007/s10862-011-9267-0 
Chen, M., \& Bargh, J. A. (1999). Consequences of Automatic Evaluation: Immediate Behavioral Predispositions to Approach or Avoid the Stimulus. Personality and Social Psychology Bulletin, 25(2), 215-224. https://doi.org/10.1177/0146167299025002007

Czar, K. A., Dahlen, E. R., Bullock, E. E., \& Nicholson, B. C. (2011). Psychopathic personality traits in relational aggression among young adults. Aggressive Behavior, 37(2), 207-214. https://doi.org/10.1002/ab.20381

Davis, M. H. (1983). Measuring individual differences in empathy: Evidence for a multidimensional approach. Journal of Personality and Social Psychology, 44(1), 113-126. https://doi.org/10.1037/0022-3514.44.1.113

De Brito, S. A., Viding, E., Kumari, V., Blackwood, N., \& Hodgins, S. (2013). Cool and Hot Executive Function Impairments in Violent Offenders with Antisocial Personality Disorder with and without Psychopathy. PLoS ONE, 8(6), e65566. https://doi.org/10.1371/journal.pone.0065566

Decety, J. (2010). The neurodevelopment of empathy in humans. Developmental Neuroscience, 32(4), 257-267. https://doi.org/10.1159/000317771

Decety, J., Michalska, K. J., Akitsuki, Y., \& Lahey, B. B. (2009). Atypical empathic responses in adolescents with aggressive conduct disorder: A functional MRI investigation. Biological Psychology, 80(2), 203-211. https://doi.org/10.1016/j.biopsycho.2008.09.004

Decety, J., \& Sommerville, J. A. (2003). Shared representations between self and other: A social cognitive neuroscience view. Trends in Cognitive Sciences, 7(12), 527-533. https://doi.org/10.1016/j.tics.2003.10.004

Eagly, A. H., \& Steffen, V. J. (1986). Gender and Aggressive Behavior. A Meta-Analytic Review of the Social Psychological Literature. Psychological Bulletin, 100(3), 309-330. 
https://doi.org/10.1037/0033-2909.100.3.309

542

Estes, W. K., \& Skinner, B. F. (1941). Some quantitative properties of anxiety. Journal of Experimental Psychology, 29(5), 390-400. https://doi.org/10.1037/h0062283

Fareri, D. S., Niznikiewicz, M. A., Lee, V. K., \& Delgado, M. R. (2012). Social network modulation of reward-related signals. Journal of Neuroscience, 32(26), 9045-9052. https://doi.org/10.1523/JNEUROSCI.0610-12.2012

Gray, J. A., \& McNaughton, N. (2000). The Neuropsychology of Anxiety: An Enquiry into the Functions of the Septo-Hippocampal System, Second Edition. Oxford University Press.

Guitart-Masip, M., Chowdhury, R., Sharot, T., Dayan, P., Duzel, E., \& Dolan, R. J. (2012a). Action controls dopaminergic enhancement of reward representations. Proceedings of the National Academy of Sciences of the United States of America, 109(19), 7511-7516. https://doi.org/10.1073/pnas.1202229109

Guitart-Masip, M., Duzel, E., Dolan, R., \& Dayan, P. (2014). Action versus valence in decision making. Trends in Cognitive Sciences, 18(4), 194-202. https://doi.org/10.1016/j.tics.2014.01.003

Guitart-Masip, M., Fuentemilla, L., Bach, D. R., Huys, Q. J. M., Dayan, P., Dolan, R. J., \& Duzel, E. (2011). Action dominates valence in anticipatory representations in the human striatum and dopaminergic midbrain. J Neurosci, 31(21), 7867-7875. https://doi.org/10.1523/JNEUROSCI.6376-10.2011

Guitart-Masip, M., Huys, Q. J. M., Fuentemilla, L., Dayan, P., Duzel, E., \& Dolan, R. J. (2012b). Go and no-go learning in reward and punishment: Interactions between affect and effect. NeuroImage, 62(1), 154-166. https://doi.org/10.1016/j.neuroimage.2012.04.024

Hershberger, W. A. (1986). An approach through the looking-glass. Animal Learning \& 
Behavior, 14(4), 443-451. https://doi.org/10.3758/BF03200092

Ickes, W., Buysse, A., Pham, H., Rivers, K., Erickson, J. R., Hancock, M., ... \& Gesn, P. R. (2000). On the difficulty of distinguishing "good" and "poor" perceivers: A social relations analysis of empathic accuracy data. Personal Relationships, 7(2), 219-234. https://doi.org/10.1111/j.1475-6811.2000.tb00013.x

Lang, P. J., Bradley, M. M., \& Cuthbert, B. N. (1990). Emotion, Attention, and the Startle Reflex. Psychological Review, 97(3), 377-395. https://doi.org/10.1037/0033-295X.97.3.377

Lengersdorff, L., Wagner, I., Lockwood, P. L., \& Lamm, C. (2020). When implicit prosociality trumps selfishness: the neural valuation system underpins more optimal choices when learning to avoid harm to others than to oneself. The Journal of Neuroscience, 40(38), 7286-7299. https://doi.org/10.1523/JNEUROSCI.0842-20.2020

Lindström, B., Haaker, J., \& Olsson, A. (2018). A common neural network differentially mediates direct and social fear learning. NeuroImage, 167, 121-129. https://doi.org/10.1016/j.neuroimage.2017.11.039

Lockwood, P. L., Apps, M. A. J., \& Chang, S. W. C. (2020). Is There a 'Social' Brain? Implementations and Algorithms. Trends in Cognitive Sciences, 24(10), P802-813. https://doi.org/10.1016/j.tics.2020.06.011

Lockwood, P. L., Apps, M. A. J., Valton, V., Viding, E., \& Roiser, J. P. (2016). Neurocomputational mechanisms of prosocial learning and links to empathy. Proceedings of the National Academy of Sciences of the United States of America, 113(35), 9763-9768. https://doi.org/10.1073/pnas.1603198113

Lorber, M. F. (2004). Psychophysiology of aggression, psychopathy, and conduct problems: a meta-analysis. Psychological Bulletin, 130(4), 531-552. https://doi.org/10.1037/0033- 
588

Lozier, L. M., Cardinale, E. M., Vanmeter, J. W., \& Marsh, A. A. (2014). Mediation of the relationship between callous-unemotional traits and proactive aggression by amygdala response to fear among children with conduct problems. JAMA Psychiatry, 71(6), 627-636. https://doi.org/10.1001/jamapsychiatry.2013.4540

Ly, V., von Borries, A. K. L., Brazil, I. A., Bulten, B. H., Cools, R., \& Roelofs, K. (2016). Reduced Transfer of Affective Value to Instrumental Behavior in Violent Offenders. Journal of Abnormal Psychology, 125(5), 657-663. https://doi.org/10.1037/abn0000166

Marsh, A. A. (2016). Understanding amygdala responsiveness to fearful expressions through the lens of psychopathy and altruism. Journal of Neuroscience Research, 94(6), 513-525. https://doi.org/10.1002/jnr.23668

Marsh, A. A. (2018). The caring continuum: Evolved hormonal and proximal mechanisms explain prosocial and antisocial extremes. Annual Review of Psychology, 70, 347-371. https://doi.org/10.1146/annurev-psych-010418-103010

Marsh, A. A., Kozak, M. N., \& Ambaby, N. (2007). Accurate identification of fear facial expression predicts prosocial behavior. Emotion, 7(2), 239-251. https://doi.org/10.1037/1528-3542.7.2.239

Miller, P. A., \& Eisenberg, N. (1988). The relation of empathy to aggressive and externalizing/antisocial behavior. Psychological Bulletin, 103(3), 324-344. https://doi.org/10.1037/0033-2909.103.3.324

Mkrtchian, A., Aylward, J., Dayan, P., Roiser, J. P., \& Robinson, O. J. (2017). Modeling avoidance in mood and anxiety disorders using reinforcement learning. Biological Psychiatry, 82(7), 532-539. https://doi.org/10.1016/j.biopsych.2017.01.017 
Mobbs, D., Yu, R., Meyer, M., Passamonti, L., Seymour, B., Calder, A. J., Schweizer, S., Frith, C. D., \& Dalgleish, T. (2009). A key role for similarity in vicarious reward. Science, 324(5929), 900. https://doi.org/10.1126/science.1170539

Murray, L., Shaw, D. S., Forbes, E. E., \& Hyde, L. W. (2017). Reward-related neural correlates of antisocial behavior and callous-unemotional traits in young men. Biological Psychiatry: Cognitive Neuroscience and Neuroimaging, 2(4), 346-354. https://doi.org/10.1016/j.bpsc.2017.01.009

Murray, L., Waller, R., \& Hyde, L. W. (2018). A systematic review examining the link between psychopathic personality traits, antisocial behavior, and neural reactivity during reward and loss processing. Personality Disorders: Theory, Research, and Treatment, 9(6), 497-509. https://doi.org/10.1037/per0000308

Reniers, R. L. E. P., Corcoran, R., Drake, R., Shryane, N. M., \& Völlm, B. A. (2011). The QCAE: A questionnaire of cognitive and affective empathy. Journal of Personality Assessment, 93(1), 84-95. https://doi.org/10.1080/00223891.2010.528484

Rescorla, R. A., \& Solomon, R. L. (1967). Two-process learning theory: relationships between pavlovian conditioning and instrumental learning. Psychological Review, 74(3), 151-182. https://doi.org/10.1037/h0024475

Rescorla, R. A., \& Wagner, A. R. (1972). A theory of Pavlovian conditioning: Variations in the effectiveness of reinforcement and nonreinforcement. In A. H. Black \& W. F. Prokasy (Eds.), Classical Conditioning II Current Research and Theory (pp. 64-99). AppletonCentury-Crofts.

Slawinski, B. L., Klump, K. L., \& Burt, A. S. (2018). No sex differences in the origins of covariation between social and physical aggression. Psychological Medicine, 49(15), 1-9. 
https://doi.org/10.1017/s0033291718003392

634

635

636

637

638

639

640

641

642

643

644

645

646

647

648

649

650

651

652

653

654

655

Solarz, A. K. (1960). Latency of instrumental responses as a function of compatibility with the meaning of eliciting verbal signs. Journal of Experimental Psychology, 59(4), 239-245. https://doi.org/10.1037/h0047274

Spielberger, C. D. (2010). State-Trait Anxiety Inventory. In The Corsini Encyclopedia of Psychology.

Sutton, R. S., \& Barto, A. G. (1998). Introduction to Reinforcement Learning. Cambridge: MIT press.

Vachon, D. D., Lynam, D. R., \& Johnson, J. A. (2014). The (non)relation between empathy and aggression: Surprising results from a meta-analysis. Psychological Bulletin, 140(3), 751773. https://doi.org/10.1037/a0035236

White, S. F., Fowler, K. A., Sinclair, S., Schechter, J. C., Majestic, C., Pine, D. S., \& Blair, R. J. R. (2014). Disrupted Expected Value Signaling in Youth With Disruptive Behavior Disorders to Environmental Reinforcers. Journal of the American Academy of Child and Adolescent Psychiatry, 53(5), 579-588. https://doi.org/10.1016/j.jaac.2013.12.023

White, S. F., Tyler, P. M., Erway, A. K., Botkin, M. L., Kolli, V., Meffert, H., Pope, K., \& Blair, J. R. (2016). Dysfunctional representation of expected value is associated with reinforcement-based decision-making deficits in adolescents with conduct problems. Journal of Child Psychology and Psychiatry, 57(8), 938-946. https://doi.org/10.1111/jcpp.12557

Zhou, Q., Valiente, C., \& Eisenberg, N. (2003). Empathy and its measurement. In S. J. Lopez \& C. R. Snyder (Eds.), Positive psychological assessment: A handbook of models and measures (pp. 269-284). American Psychological Association. 


\title{
Modeling variation in empathic sensitivity using go/no-go social reinforcement learning
}

\author{
Supplementary Materials
}

Katherine O'Connell*, Marissa Walsh, Brandon Padgett, Sarah Connell \& Abigail A. Marsh 


\section{Supplementary Text}

Immediately following completion of the task subjects were directed to complete a computerized survey, which first asked them to rate for each condition how motivated they were to earn additional money (1-not at all motivated, 7-extremely motivated), how motivated they were to avoid losing money (1-not at all motivated, 7-extremely motivated), and their overall effort exerted (1-no effort, 7-maximal effort).

Results of a 3 condition (self, social, no one) x 2 valence (win, avoid loss) repeated measures ANOVA on motivation revealed a significant main effect of condition $(F(2,112)=43.53$, $\left.\mathrm{p}<.001, \eta \mathrm{p}^{2}=.44,95 \% \mathrm{CI}=[.30, .54]\right)$ driven by reduced reported motivation during the no one condition (Supplementary Table 2). Subjects reported similarly moderate-to-high motivation to win and avoid losing money during both the self and social conditions. No significant main effect of valence $(F(1,56)=2.30, p=.135)$ nor a condition $x$ valence interaction $(F(2,112)=0.38, p=.686)$ emerged. Ratings of effort also revealed a main effect of condition $(F(2,112)=22.17, p<.001$, $\left.\eta \mathrm{p}^{2}=.28,95 \% \mathrm{CI}=[.14, .40]\right)$ such that effort was lowest during the no one condition, but showed no difference between the self and social conditions $(\mathrm{p}>.999)$. 


\section{Supplementary Tables}

Supplementary Table 1. Characteristics of the final sample

\begin{tabular}{|c|c|c|}
\hline & & Min-Max \\
\hline $\mathrm{N}$ & 61 & \\
\hline Age, $M(S D)$ & $23.0(6.9)$ & $18-49$ \\
\hline Male/Female ( $\%$ Female $)$ & $21 / 40(66 \%)$ & \\
\hline \multicolumn{3}{|l|}{ Race } \\
\hline White/ Caucasian & $32(53 \%)$ & \\
\hline Black/African-American & $8(13 \%)$ & \\
\hline Asian & $15(25 \%)$ & \\
\hline Other & $5(8 \%)$ & \\
\hline Trait Anxiety STAI-Y2, $M(S D)$ & $43.7(12.4)$ & $23-71$ \\
\hline Interpersonal Reactivity Index (IRI) & $68.5(12.9)$ & $28-91$ \\
\hline Perspective Taking, $M(S D)$ & $18.5(4.7)$ & $5-27$ \\
\hline Fantasy, $M(S D)$ & $17.4(5.3)$ & $7-26$ \\
\hline Empathic Concern, $M(S D)$ & $20.9(4.6)$ & $7-28$ \\
\hline Personal Distress, $M(S D)$ & $11.7(5.3)$ & $1-25$ \\
\hline \multicolumn{3}{|c|}{ Subtypes of Antisocial Behavior (STAB) } \\
\hline Physical Aggression, $M(S D)$ & $18.0(4.5)$ & $12-36$ \\
\hline Social Aggression, $M(S D)$ & $21.5(4.0)$ & $15-30$ \\
\hline Rule-Breaking, $M(S D)$ & $12.4(2.2)$ & $11-21$ \\
\hline
\end{tabular}


Supplementary Table 2. Self-reported motivation and effort

\begin{tabular}{lccc}
\hline & Self & Social & No One \\
\hline Motivated to win & $5.07[4.71,5.44]$ & $5.09[4.69,5.49]$ & $3.54[3.13,3.96]$ \\
Motivated to avoid loss & $4.95[4.58,5.32]$ & $5.05[4.64,5.46]$ & $3.39[2.96,3.81]$ \\
Effort exerted & $3.46[3.24,3.68]$ & $3.46[3.22,3.70]$ & $2.74[2.50,2.98]$
\end{tabular}

Note: Four subjects did not complete motivation and effort ratings. 95\% CI of the mean provided in brackets. 
Supplementary Table 3. Model parameters during social learning in relation to antisociality

\begin{tabular}{|c|c|c|c|c|c|c|c|c|}
\hline & $\beta_{\text {win }}$ & $\beta_{\text {loss }}$ & $\pi_{\text {win }}$ & $\pi_{\text {loss }}$ & $\alpha$ & $\mathrm{x}$ & $\mathrm{b}$ & $\mathrm{q}$ \\
\hline $\begin{array}{l}\text { STAB } \\
\text { Social }\end{array}$ & $-.25(.055)$ & $\mathbf{- . 2 7 ~ ( . 0 3 4 )}$ & $-.07(.622)$ & $-.07(.573)$ & $\mathbf{. 2 9}(\mathbf{. 0 2 5})$ & $-.23(.076)$ & $-.13(.329)$ & $.18(.152)$ \\
\hline
\end{tabular}

Note: Cells show Pearson r values. The perm in parentheses is uncorrected for multiple comparisons.

Supplementary Table 4. Model parameters during social learning in relation to trait empathy

\begin{tabular}{|l|c|c|c|c|c|c|c|c|}
\hline & $\beta_{\text {win }}$ & $\beta_{\text {loss }}$ & $\pi_{\text {win }}$ & $\pi_{\text {loss }}$ & $\alpha$ & $\mathrm{x}$ & $\mathrm{b}$ & $\mathrm{q}$ \\
\hline IRI PT & $.08(.548)$ & $.11(.375)$ & $.21(.098)$ & $-.01(.900)$ & $-.12(.339)$ & $.01(.921)$ & $-.06(.672)$ & $.11(.403)$ \\
\hline IRI F & $.03(.831)$ & $-.04(.780)$ & $.16(.210)$ & $-.01(.929)$ & $-.14(.286)$ & $-.09(.470)$ & $-.16(.226)$ & $.28(.028)$ \\
\hline IRI EC & $.10(.455)$ & $.14(.297)$ & $.02(.886)$ & $.09(.509)$ & $-.22(.098)$ & $.07(.578)$ & $.00(.992)$ & $.10(.459)$ \\
\hline IRI PD & $.13(.333)$ & $.03(.782)$ & $-.10(.448)$ & $.15(.262)$ & $-.14(.283)$ & $-.13(.317)$ & $.17(.195)$ & $-.02(.891)$ \\
\hline IRI Total & $.13(.320)$ & $.09(.488)$ & $.11(.386)$ & $.08(.534)$ & $-.24(.070)$ & $-.06(.642)$ & $-.02(.911)$ & $.18(.159)$ \\
\hline
\end{tabular}

Note: Cells show Pearson $\mathrm{r}$ values. The $\mathrm{p}_{\mathrm{perm}}$ in parentheses is uncorrected for multiple comparisons.

Supplementary Table 5. Model parameters in relation to trait anxiety

\begin{tabular}{|l|c|c|c|c|c|c|c|c|c|}
\hline & \multicolumn{7}{|c|}{ Social } \\
\hline & $\beta_{\text {win }}$ & $\beta_{\text {loss }}$ & $\pi_{\text {win }}$ & $\pi_{\text {loss }}$ & $\alpha$ & $\mathrm{x}$ & $\mathrm{b}$ & $\mathrm{q}$ \\
\hline STAI-Y2 & $.04(.762)$ & $-.01(.921)$ & $.06(.663)$ & $.10(.480)$ & $-.07(.617)$ & $-.15(.245)$ & $.00(.997)$ & $.07(.579)$ \\
\hline & \multicolumn{7}{|c|}{ Self } \\
\hline & $\beta_{\text {win }}$ & $\beta_{\text {loss }}$ & $\pi_{\text {win }}$ & $\pi_{\text {loss }}$ & $\alpha$ & $\mathrm{x}$ & $\mathrm{b}$ & $\mathrm{q}$ \\
\hline STAI-Y2 & $-.13(.318)$ & $-.13(.309)$ & $-.11(.389)$ & $\mathbf{. 2 6}(\mathbf{. 0 4 5})$ & $-.22(.090)$ & $.08(.550)$ & $.22(.085)$ & $-.15(.240)$ \\
\hline
\end{tabular}


Note: Cells show Pearson $\mathrm{r}$ values. The $\mathrm{p}_{\text {perm }}$ in parentheses is uncorrected for multiple comparisons.

\section{Supplementary Figures}

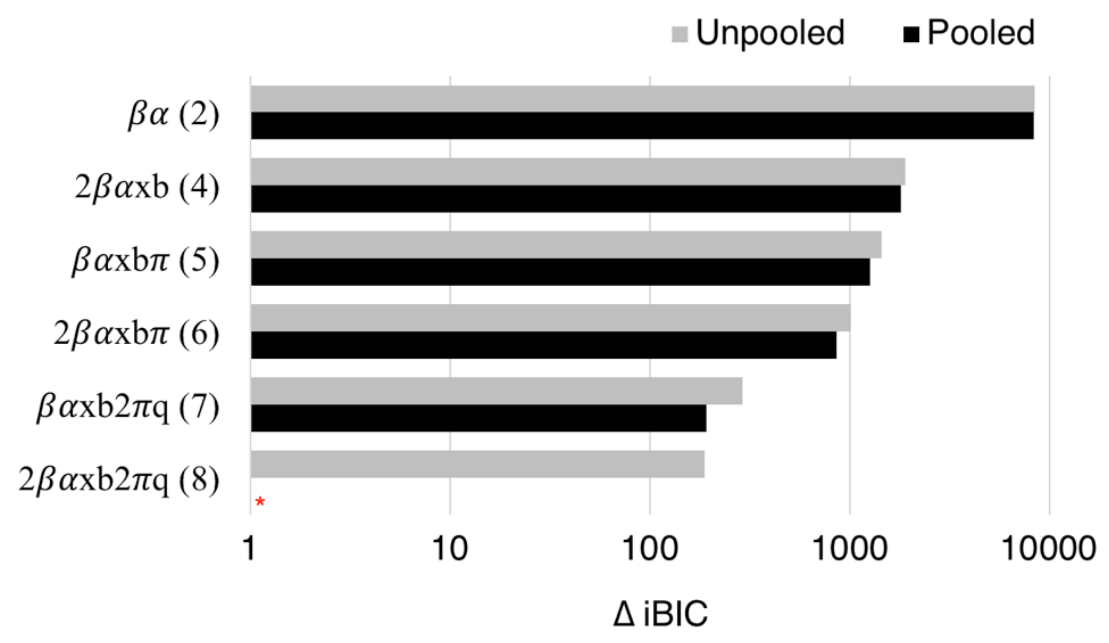

Supplementary Fig. 1 Model comparison. An adapted Rescorla-Wagner model with 8

parameters outperformed other models and the pooled distribution across conditions fit better than the unpooled distributions. Note the $\mathrm{x}$-axis is on a logarithmic scale. The red asterisk indicates the best fitting model (Pooled distribution for $2 \beta \alpha \mathrm{xb} 2 \pi \mathrm{q}$; $\mathrm{BIC}=4.62 \times 10^{4}$ ) 

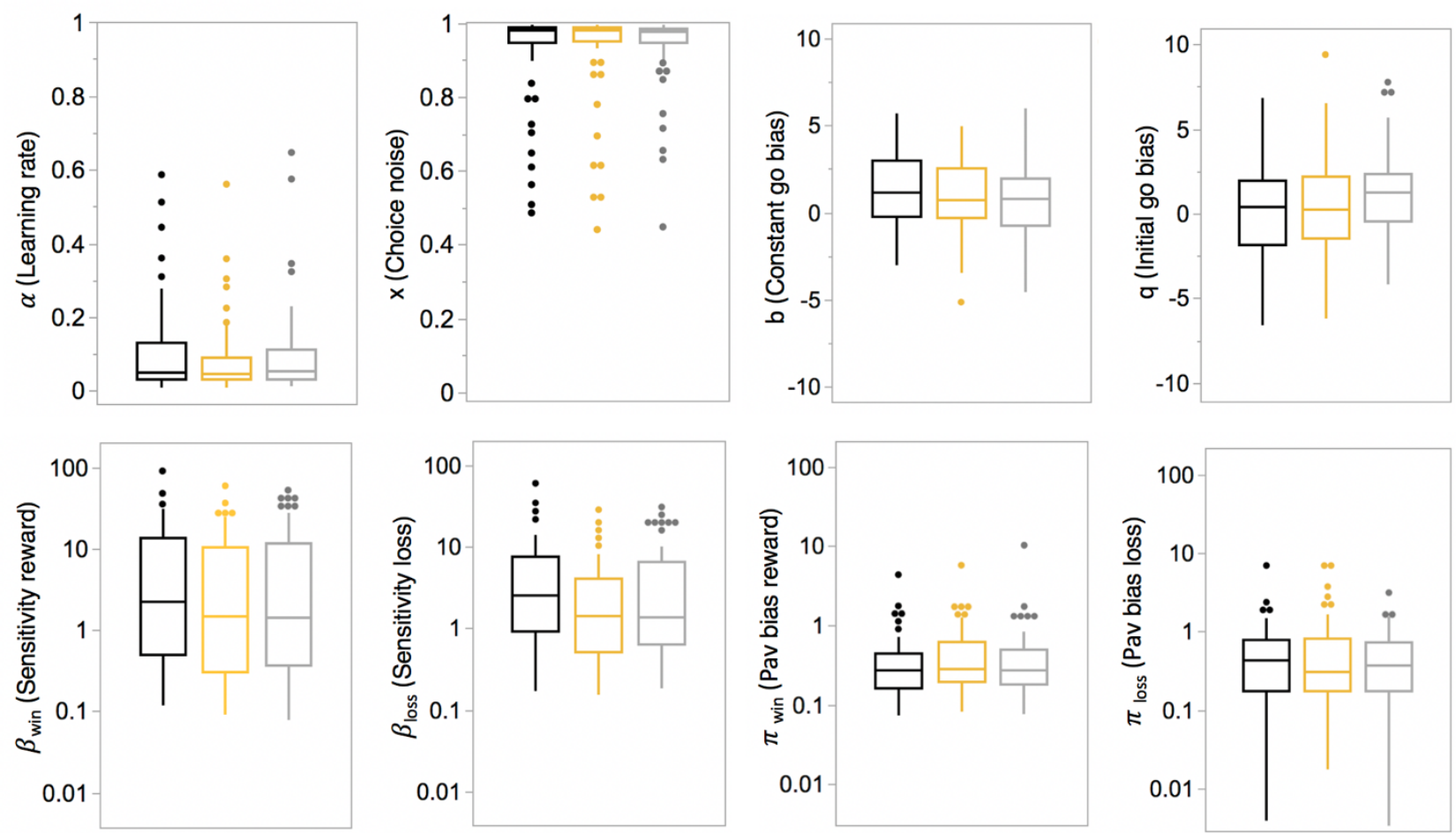

Supplementary Fig. 2 Transformed model parameters by condition. Note the logarithmic scale for the $\beta$ and $\pi$ parameters. Self $=$ black, social $=$ orange, no one $=$ light gray 

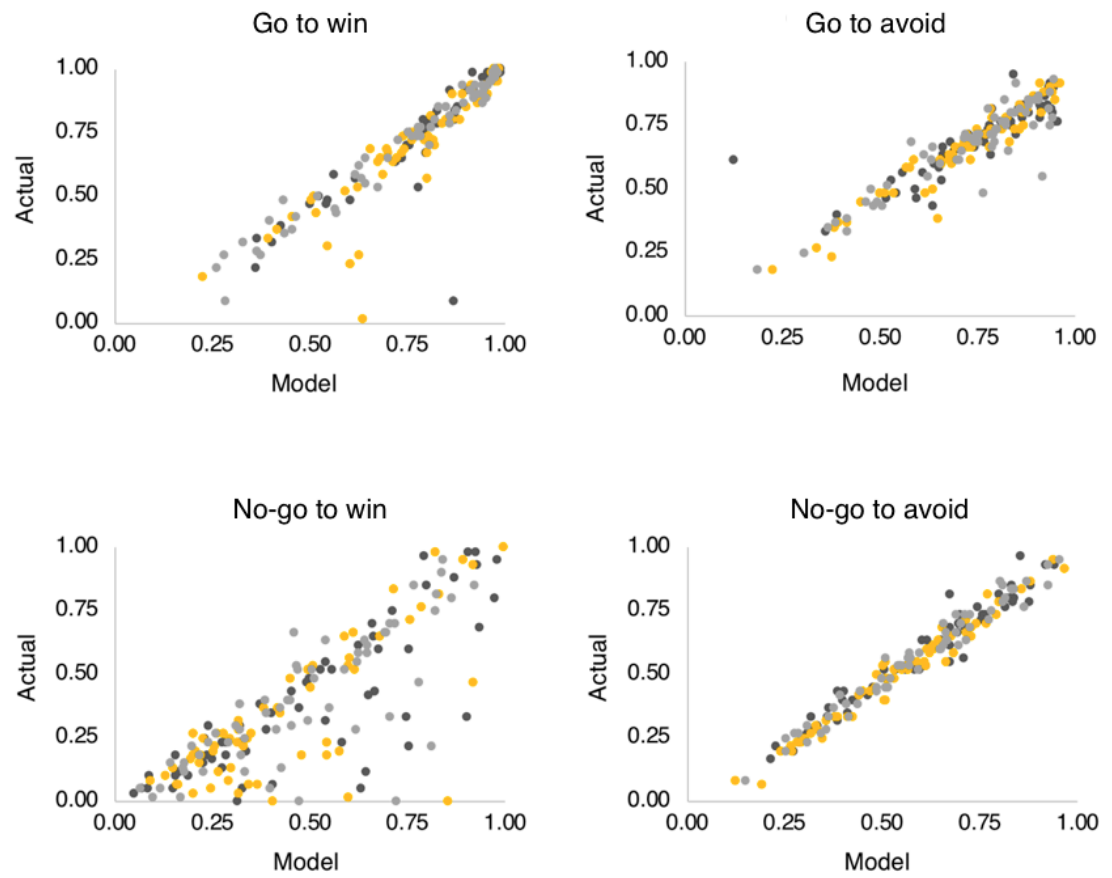

Supplementary Fig. 3 Posterior predictive model mean accuracy plotted by subjects' actual mean accuracy. Simulated mean accuracy (x-axis) in relation to actual mean accuracy (y-axis) for each subject. The model performs well but can overestimate accuracy, especially in the no-go to win trial type 


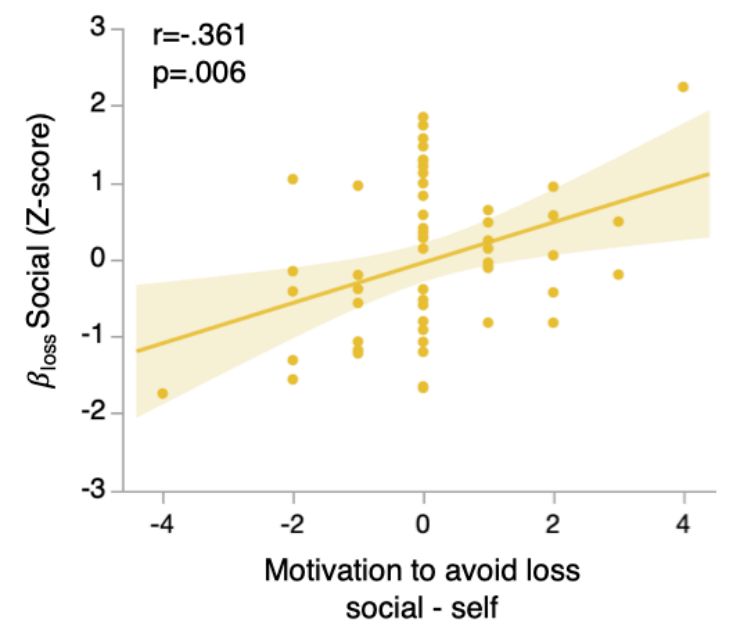

Supplementary Fig. 4 Correlation between motivation to avoid loss for the partner and $\beta_{\text {loss }}$ sensitivity. Pearson correlation between z-scored $\beta_{\text {loss }}$ sensitivity obtained during the social condition and reported motivation to avoid loss in the social relative to self condition 